\title{
Andre Gide - Edmond Jaloux, Correspondance,
} 1896-1950

\section{Emanuele Kanceff}

\section{Q OpenEdition \\ 1 Journals}

\section{Edizione digitale}

URL: http://journals.openedition.org/studifrancesi/35107

DOI: $10.4000 /$ studifrancesi.35107

ISSN: 2421-5856

\section{Editore}

Rosenberg \& Sellier

\section{Edizione cartacea}

Data di pubblicazione: 1 novembre 2005

Paginazione: 446-447

ISSN: 0039-2944

\section{Notizia bibliografica digitale}

Emanuele Kanceff, «Andre Gide - Edmond Jaloux, Correspondance, 1896-1950», Studi Francesi [Online], 146 (XLIX | II) | 2005, online dal 30 novembre 2015, consultato il 18 avril 2021. URL: http://

journals.openedition.org/studifrancesi/35107 ; DOI: https://doi.org/10.4000/studifrancesi.35107 


\title{
Andre Gide - Edmond Jaloux, Correspondance, 1896-1950
}

\author{
Emanuele Kanceff
}

\section{NOTIZIA}

ANDRE GIDE - EDMOND JALOUX, Correspondance, 1896-1950. Édition établie, présentée et annotée par Pierre LACHASSE, Lyon, Presses Universitaires, 2004, pp. 416.

1 Giovedì 23 aprile 1896, nella sua stanza di via dei Tonneliers a Marsiglia, un giovane poeta di 18 anni, il quale aveva pubblicato a spese d'autore una raccolta di versi simbolistici, ricevette la visita inattesa di André Gide, di otto anni più vecchio di lui. Tra Edmond Jaloux e l'autore di Paludes e del Voyage d'Urien di cui egli lesse le opere come fossero un breviario da cui attingere per dare un senso alla sua vita, e al suo cammino letterario, è l'inizio di una lunga amicizia che, senza essere sia per l'uno che per l'altro messa in primo piano, durerà attraverso alti e bassi fino alla loro morte. La corrispondenza, inaugurata da questo primo incontro, costituisce per il più giovane una vera iniziazione morale e intellettuale, segnata dalle rinnovate letture delle opere del più anziano il «Récit de Ménalque», Les Nourritures terrestres e L'Immoraliste di cui è uno dei primi a capirlo poi attraverso la scrittura dei suoi primi testi in prosa che lui tremante sottopone al suo maestro, infine attraverso la febbrile attesa delle sue visite che lui si augura diventino sempre più frequenti.

2 Questa appassionata amicizia del poeta apprendista per lo scrittore già conosciuto da parte di una élite, potrebbe sembrare sproporzionata, ma Gide, il quale prova una grande simpatia per l'entusiasmo del neofita, sperimenta la forza della sua infuenza letteraria aiutandolo a scoprirsi romanziere.

3 Queste lettere, per la gran parte inedite, scoprono gli inizi di un'avventura intellettuale e mostrano che l'educazione di uno spirito non consiste a farlo uguale a se stesso, ma a sviluppare una profonda singolarità, una sua «idiosincrasia» direbbe Gide. Queste lettere ridanno vita anche a Edmond Jaloux, alla sua abbondante opera, ingiustamente 
dimenticata, che lo condusse all'Accademia e di cui il magistero, notoriamente esercitato tramite le Nouvelles littéraires, fece di lui il critico più temuto del suo tempo.

Tra le 112 lettere che l'autore ha pubblicato, 74 sono di Gide e 37 di Jaloux, alle quali bisogna aggiungerne una collettiva firmata A. Eustache, Albert-J. Brandenburg e Edmond Jaloux (lettera 5). Esse sono conservate presso la Biblioteca letterale JacquesFoucet, ad eccezione di tre: la lettera 82, già pubbliata nel Baag n. 66, nell'aprile 1985; la lettera 91, apparsa ne Lse Nouvelles littéraires del 14 aprile 1928 e la lettera 33, di cui l'autore conosce solo un breve frammento apparso nella cronaca bibliografica del Baag n. 135-136, del luglio-ottobre 2002. I quattro frammenti di lettere citate o riassunte nel Baag n. 17, dell'ottobre 1972, p. 12, e pubblicate nei Beaux Livres et Autographes in occasione dell'asta Drouot del 12, 13 e 19 febbraio 1959 non sono utilizzabili, anche se si può senz'altro comprendere che si tratta per tre di loro di scambi preparando quello che si è deciso di chiamare il banchetto Jaloux (1909). Il quarto frammento è evidentemente tratto da una lettera di Henri de Régnier a Gide dove si tratta la questione del premio Vie Heureuse attribuito a Jaloux.

5 L'autore ha corretto gli errori di ortografia dovuti alla disattenzione degli scrittori, indicando la modifica nel testo, tra parentesi quadre. Solo in casi rari l'autore ha mantenuto l'errore segnalandolo con un «sic» in nota. Per quanto concerne i nomi propri russi, ne ha uniformato la trascrizione secondo l'uso attuale (per esempio, Dostoievski al posto di Dostoïewsky). Per la stessa ragione, ha alleggerito il testo dagli abbondanti trattini, conservando solo quelli che permettono di far respirare la frase, come è l'uso coretto come segni di punteggiatura.

6 Visto che sia la figura che i libri di Edmond Jaloux non sono conosciuti se non dagli specialisti nel ramo, visto anche che non esiste nessuna versione moderna delle sue opere, l'autore ha riservato loro gran parte dell'introduzione e dell'apparato critico, considerando che i numerosissimi lavori di Gide permettono ai lettori curiosi di colmare con facilità le loro eventuali lacune. Per lo stesso motivo ha considerato come conosciuti i numerosi scrittori che circondano Gide a L'Ermitage, poi a La NRF e non ha ritenuto utile dilungarsi in molte note e spiegazioni. Dopo le lettere, l'estensore pubblica numerosi testi che chiariscono, molto più di un semplice commento, le relazioni dei due scrittori. In Appendice A, ha raccolto l'insieme degli scritti, a volte ridondanti, dedicati da Jaloux a Gide e alla sua opera, di cui una parte essenziale è apparsa in alcuni periodici; l'inventario non è senza dubbio esaustivo, perché Lachasse è ben lontano dall'aver potuto spogliare la grande abbondanza di contributi di Jaloux apparsi nei giornali e nelle riviste apparse in Francia e all'estero nel corso di più di mezzo secolo. In Appendice B ha riunito un breve testo narrativo di Jaloux, la di cui lettura permette di apprezzare meglio i suoi inizi come scrittore, poi delle recensioni critiche di Henri Ghéon e di Gide dei suoi primi due romanzi. Il testo di Gide dedicato a L'Agonie de l'Amour è inedito dopo la sua pubblicazione, non firmata, ne «L'Ermitage» nel marzo 1900. In Appendice $C$ Lachasse ci mostra la lista dei numerosi libri pubblicati da Edmond Jaloux, una lista però non completa di tutti i periodici con i quali lui ha collaborato e qualche elemento di bibliografia critica. L'autore tiene a ringraziare Catherine Gide, che ha dato l'autorizzazione alla consultazione e alla pubblicazione di questa corrispondenza, la Società delle Gens de Lettres de France, legatari di Edmond Jaloux, Claude Martin, Pierre Masson, Pascal Mercier e Thomas Reisen, alcuni per l'incoraggiamento e gli altri per le pronte risposte date alle domande, la Biblioteca letteraria Jacque-Doucet, la Bibliothèque de l'Arsenal e la Bibliothèque Méjane di Aix- 
en-Provence che ha dato il permesso di pubblicare la fotografia di Edmond Jaloux datata ottobre 1897, quando aveva 19 anni, all'epoca della sua intensa relazione intellettuale con André Gide.

7 Questa edizione è arricchita di un indice dei nomi molto esteso e diligente, strumento che è sempre auspicabile ma nel presente caso indispensabile, trattandosi di un reperto di documenti epistolari ricco e circostanziato, in cui il riferimento alla storia o alla cronaca è un elemento ricorrente. 